\title{
Spin-orbit coupling in three-orbital Kanamori impurity model and its relevance for transition-metal oxides
}

\author{
Alen Horvat, ${ }^{1}$ Rok Žitko, ${ }^{1,2}$ and Jernej Mravlje ${ }^{1}$ \\ ${ }^{1}$ Jožef Stefan Institute, Jamova 39, Ljubljana, Slovenia \\ ${ }^{2}$ Faculty of Mathematics and Physics, University of Ljubljana, Jadranska 19, Ljubljana, Slovenia
}

\begin{abstract}
We investigate the effects of the spin-orbit coupling (SOC) in a three-orbital impurity model with Kanamori interaction using the numerical renormalization group method. We focus on the impurity occupancy $N_{d}=2$ relevant to the dynamical mean-field theory studies of Hund's metals. Depending on the strength of SOC $\lambda$ we identify three regimes: usual Hund's impurity for $|\lambda|<\lambda_{c}$, van-Vleck non-magnetic impurity for $\lambda>\lambda_{c}$, and a $J=2$ impurity for $\lambda<-\lambda_{c}$. They all correspond to a Fermi liquid but with very different quasiparticle phase shifts and different physical properties. The crossover between these regimes is controlled by an emergent scale, the orbital Kondo temperature, $\lambda_{c}=T_{K}^{\text {orb }}$ that drops with increasing interaction strength. This implies that oxides with strong electronic correlations are more prone to the effects of the spin-orbit coupling.
\end{abstract}

\section{INTRODUCTION}

At energies relevant to solid-state physics, the relativistic effects manifest most prominently as the spin-orbit coupling (SOC) term in the Hamiltonian, $H_{\mathrm{SOC}}=\lambda \mathbf{l} \cdot \mathbf{s}$. The alignment of spin and orbital degrees of freedom has several effects: at the level of noninteracting electronic structure, it leads to the lifting of the band degeneracies and can induce a change of topology in the momentum space, a topic widely discussed today; in atoms, the SOC leads to the third Hund's rule; in magnetism, it leads to the spin-anisotropies. The strength of the SOC increases with atomic number, with typical values of $50 \mathrm{meV}$ for $3 \mathrm{~d}$ oxides, $0.1-0.2 \mathrm{eV}$ for $4 \mathrm{~d}$ oxides, and about $0.4 \mathrm{eV}$ for $5 \mathrm{~d}$ oxides. Recently the strong effects of the SOC have been carefully investigated in $5 \mathrm{~d}$ oxides, in particular irridates with 5 electrons occupying the d-shell, where the energy of the $j=3 / 2$ states is lowered leading to a half-filled $j=1 / 2$ band and the associated occurence of the Mott transition $^{1-3}$.

The situation in $4 \mathrm{~d}$ oxides with an intermediate strength of the SOC, notably ruthenates, is more nuanced. On one hand, in band-structure calculations ${ }^{4,5}$ and in spin-sensitive photoemission ${ }^{6}$ the effects of the SOC have been clearly observed. On the other hand, within the dynamical-mean field theory (DMFT) approach the ruthenates have been widely and succesfully ${ }^{7-12}$ discussed as Hund's metals (compounds in which the coherence scale is suppressed by the Hund's interaction $J_{H}$ ) without taking the effects of $\lambda$ into account at all. In ruthenates, the SOC is $0.1-0.2 \mathrm{eV}$, which is similar to $J_{H}$ that is about $0.3-0.4 \mathrm{eV}$. This prompts the question to what scale $T_{0}$ must one compare the strength of the spin-orbit coupling to determine whether its effects are important. Furthermore, for four electrons in the $t_{2 g}$ shell strong enough SOC leads to a non-magnetic $J=0$ van-Vleck insulator regime ${ }^{13-17}$, but the threshold SOC strength remains to be quantified. Finally, there are also important open qualitative questions. A very recent model DMFT work found that the SOC substantially modifies the behavior and leads to an interesting
non-Fermi-liquid behavior dubbed the $J$-freezing ${ }^{18}$. In the absence of SOC, Hund's metals are Fermi liquids which follows from the physics of the underlying impurity model $^{12}$. The three-orbital impurity model with SOC has not been explored so far and the nature of its low-energy fixed points is unknown.

In the present work we investigate these questions within a three-orbital Kanamori impurity model with spin-orbit coupling at occupancies $N_{d}=2$ and 4 which are relevant to Hund's metals. To solve the model, we have implemented a numerical renormalization group (NRG) code exploiting the conservation of total angular momentum $J$ to keep the computational cost manageable. The ground-state of the impurity problem is found to always be a Fermi liquid, but characterizing it in terms of the quasiparticle phase-shifts one can distinguish three regimes: a Hund's metal for $|\lambda|<\lambda_{c}$, a non-magnetic van-Vleck regime for $\lambda>\lambda_{c}$, and a $j=3 / 2$ metal for $\lambda<-\lambda_{c}$. We find that the crossover scale $\lambda_{c}$ is given not by a bare parameter, but rather by an emergent scale: the orbital Kondo temperature. We also calculated the impurity spectral function which is found to exhibit characterically different shapes in the three regimes. This not only has implications for the physics of oxides described within the DMFT but could also be directly observed in the tunneling spectra of Hund's impurities adsorbed on metal surfaces ${ }^{19-21}$.

\section{MODEL}

We consider a three-orbital impurity model $H=$ $H_{\text {band }}+H_{\text {hyb }}+H_{\text {imp }}$ with the Kanamori Coulomb interaction and the spin-orbit coupling on the impurity site:

$$
\begin{aligned}
H_{\mathrm{imp}}= & \frac{1}{2}\left(U-3 J_{\mathrm{H}}\right) N_{d}\left(N_{d}-1\right)- \\
& -2 J_{\mathrm{H}} \mathbf{S}^{2}-\frac{J_{\mathrm{H}}}{2} \mathbf{L}^{2}+\epsilon N_{d}+H_{\mathrm{ls}} .
\end{aligned}
$$

$U, J_{\mathrm{H}}$ are the on-site Hubbard repulsion and Hund's coupling, respectively. $N_{d}=\sum_{m} n_{m}, \mathbf{S}=\sum_{m} \mathbf{s}_{m}, \mathbf{L}=$ 
Table I. Spin-orbit coupling $\lambda$, total angular momentum $J$ and occupancy of the $p$ and $t_{2 g}$ orbitals.

\begin{tabular}{c|c}
$\lambda<0$ & $\lambda>0$ \\
\hline$J=L+S$ & $J=|L-S|$ \\
$p^{4}, d^{2}$ & $p^{2}, d^{4}$ \\
molybdates, chromates & ruthenates
\end{tabular}

$\sum_{m} \mathbf{l}_{m}$ are impurity total charge, spin and orbital momentum operators, and $n_{m}, \mathbf{s}_{m}, \mathbf{l}_{m}$ are the occupancy, spin, and orbital moment in orbital $m$. $\epsilon$ sets the occupancy of the impurity. We take the impurity to be coupled to a flat conduction band with the density of states $\rho=1 / 2 D=1 / 2, D=1$ being the half band-width, described by $H_{\text {band }}$. $H_{\text {hyb }}=\sum_{k, j, m} V_{k} c_{k j m}^{\dagger} d_{j m}+$ h.c. is the hybridization. The spin-orbit coupling $H_{l s}$ can be written in the spherical orbital basis (with $m=-1,0,1$ being the eigenstates of $l_{z}$ for $l=1$ ) as $H_{l s}=H_{l s}^{z}+H_{l s}^{x y}$ with

$$
\begin{array}{r}
H_{l s}^{z}=\frac{\lambda}{2} \sum_{m=-l}^{l} m\left(d_{m \uparrow}^{\dagger} d_{m \uparrow}-d_{m \downarrow}^{\dagger} d_{m \downarrow}\right), \\
H_{l s}^{x y}=\frac{\lambda}{2} \sum_{m=-l}^{l-1} \sqrt{(l-m)(l+m+1)} \times \\
\quad \times\left(d_{m+1 \downarrow}^{\dagger} d_{m \uparrow}+d_{m \uparrow}^{\dagger} d_{m+1 \downarrow}\right) .
\end{array}
$$

In this study we consider the model at the impurity occupancies $N_{d}=2$ and 4 . The spin-orbit operator changes sign upon a particle-hole transformation. Hence, an impurity occupied by two electrons and $\operatorname{SOC} \lambda$ is equivalent to an impurity occupied by four electrons (two holes) and SOC $-\lambda$. In our discussion we focus on the case with fixed occupancy $N_{d}=2$ and change the sign of the SOC $\lambda$ to account for the case with $N_{d}=4$. In transitionmetal oxides with $t_{2 g}$ valence orbitals the traditional 3 rd Hund's rule is inverted due to the TP correspondence which has to do with the fact that the matrix elements of $l=2$ within the $t_{2 g}$ subspace are the same as these of the $l=1$ operators (within the $p$ subspace) but with inverted $\operatorname{sign}^{22} \cdot \lambda>0$ that favors small values of $J^{2}$ is relevant to the physics of more than half-filled $t_{2 g}$ shell of $d^{4}$ oxides, such as ruthenates, whereas $\lambda<0$ that favors large values of $J^{2}$ is relevant to the less than half-filled $d^{2}$ oxides, such as molybdates, as summarized in Table I.

\section{METHOD}

We solve the impurity problem using numerical renormalization group (NRG) solver. ${ }^{23,24}$ We take into account the conservation of charge and total angular momentum to reduce the computational cost. The impact of the exponential growth of the Hilbert space depends on the NRG discretization parameter $\Lambda$. In our calculations we used $\Lambda=10$. The effect of quite large Lambda was reduced by using twist averaging over $N_{z}=8$ interleaved discretization grids ${ }^{25-27}$. In the diagonalization all the states with $E<E_{\text {keep }}=10$ are kept. We additionally limit the total number of kept states to 6000 (due to memory constraints), a restriction that is more stringent than the former one only in the first few iterations. To calculate the spectral functions we use the complete Fock space approach $^{28}$.

\section{RESULTS}

We first discuss the influence of the spin-orbit coupling on the thermodynamic expectation values. In Fig. 1(a) we display the temperature evolution of the effective local moment evaluated as $\chi_{J} T$, where $\chi_{J}$ is the impurity contribution to the total angular moment susceptibility. Panel (b) shows the impurity contribution to entropy and panel (c) shows the total angular momentum at the impurity site $\left\langle J^{2}\right\rangle$. Throughout the text the parameters are $U=3.2, J_{\mathrm{H}}=0.4$, and $\Gamma=\pi \rho_{0} V^{2}=0.05$.

Consider the $\lambda=0$ case first. With decreasing temperature $T$, after the charge fluctuations are frozen out (above temperatures shown) the model enters into the local-moment regime with a 9 -fold degenerate $L=1, S=$ 1 multiplet characterized by a plateau in the entropy at a value of $\log (9)$ and with $\chi_{J} T \approx\left\langle J^{2}\right\rangle / 3$ where $\left\langle J^{2}\right\rangle=\left\langle L^{2}\right\rangle+\left\langle S^{2}\right\rangle=4$. (This result follows also in the $J$ basis, $\left\langle J^{2}\right\rangle=(6 \cdot 5+2 \cdot 3+0 \cdot 1) / 15=4$. $)$ On further lowering the temperature, the local moment is progressively screened and becomes small below a low (Kondo) temperature.

The inset to Fig. 1 separately displays for the $\lambda=0$ case the local spin moment $\chi_{S} T$ and the orbital angular moment $\chi_{L} T$ evaluated respectively from spin and orbital susceptibilities. As discussed in earlier work ${ }^{29-31}$ the screening of the spin moment occurs at a lower temperature $T_{K}^{\mathrm{spin}}$ than the one for the orbital moment $T_{K}^{\text {orb }}$ (the two Kondo temperatures are indicated by the two vertical lines and differ by about an order of magnitude). The initial drop of $\chi_{J} T$ and the associated suppression of the impurity contribution to entropy, seen in Fig. 1(b), thus comes mainly from the quenching of the orbital degrees of freedom.

Turning on the spin-orbit interaction has a strong effect with markedly different behavior in the cases of the positive (dashed lines) and negative (plain lines) values of $\lambda$. For $\lambda<0$, the SOC tends to align the spin and the orbital moment to a state of a large total angular moment $J=L+S$. The expectation value of $J^{2}$ tends to $6=2(2+1)$, and $\chi_{J} T$ approaches 2 in the hightemperature local-moment regime when the temperature is lowered to $T<|\lambda|$. On cooling down further, the local moment is screened; the corresponding Kondo temperature is found to diminish as $|\lambda|$ increases. In the temperature window $T_{K}<T<|\lambda|$, the impurity contribution to entropy shows a clear plateau at $\log 5$ revealing the degeneracy of the $J=2$ local moment. For intermediate strenghts of $|\lambda|$ one sees first a plateau at the $\log 9$ $\mathrm{S}=\mathrm{L}=1$ manifold and then a crossover to the $\log 5$ value 

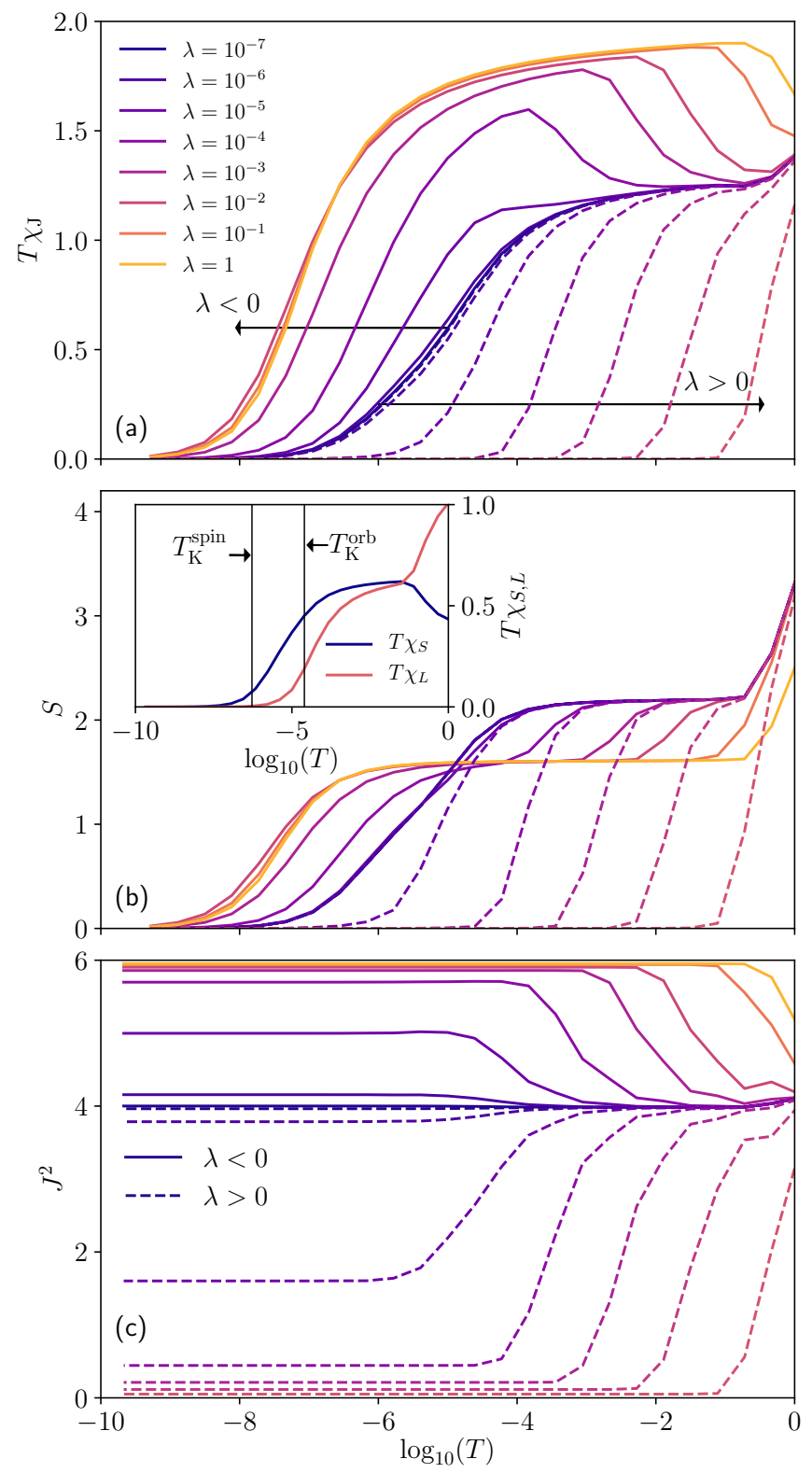

Figure 1. (a) Effective local moment. Inset: effective spin and orbit moment for $\lambda=0$. (b) Impurity entropy. (c) Expectation value $\left\langle J^{2}\right\rangle$. Solid (dashed) lines denote results for $\lambda<0(\lambda>0)$.

when the temperature drops to a value $T<|\lambda|$.

For $\lambda>0$, the SOC tends to anti-align the spin and the orbital moments which leads to a non-degenerate $J=0$ atomic ground state. This is a peculiar "no-impurity" regime of an impurity problem: there are no internal degrees of freedom at the impurity and the conduction electrons experience only potential scattering. It turns out that this atomic consideration describes the numerical results well, provided that $\lambda$ is significantly larger than the Kondo temperature itself.

We now turn to $\left\langle J^{2}\right\rangle$ shown in Fig. 1(c). For large $\lambda>0$, as soon as temperature drops below $\lambda,\left\langle J^{2}\right\rangle$ rapidly approaches a very small value. At the same temperature, the entropy and $\chi T$ also drop rapidly to 0 , as seen in panels (b) and (a). Notice the distinct behavior of $\left\langle J^{2}\right\rangle$ and $\chi_{J} T$ for positive and negative $\lambda$. In the latter case, one has a large atomic moment that is screened by the quantum fluctuations on cooling below the Kondo temperature whereas in the former case the atomic moment is not present to start with. Or, thinking in terms of the temperature dependence, for $\lambda>0$ the suppression of $\chi_{J} T$ and $\left\langle J^{2}\right\rangle$ occurs at the same scale $T \approx \lambda$ : the process is atomic and does not involve the conduction electrons.

The evolution of key quantities as a function of $\lambda$, shown in Fig. 2, serves to delineate the different regimes. In Fig. 2(a) we present $T_{K}$ defined as the temperature at which $\chi_{J} T$ drops below 0.07. For $\lambda<0$ (left from centre) we observe a reduction of $T_{K}$ with increasing $|\lambda|$. The lowering of the Kondo temperature is due to the formation of a larger moment combined with the splitting of the multiplets that leads to a suppression of the Kondo coupling strength. From the Schrieffer-Wolff transformation one finds that in the limit $|\lambda| \ll J_{H}, U$ the Kondo couplings are proportional to $1 /\left(c_{0}-\lambda\right), c_{0}>0$. For $\lambda>0$ (right from centre) the opposite behavior is seen. For large $\lambda, T_{K} \approx \lambda^{32}$. A very similar dependence on $\lambda$ is found also in the zero-temperature total angular momentum susceptibility $\chi_{J}$ that is shown in Fig. 2(b) along the expectation value $\left\langle J^{2}\right\rangle$. The evolution of $T_{K}$ as a function of the SOC $\lambda$ is indicative of the one found for the inverse of mass enhancement $Z$, $T_{K} \sim Z=\left.(1-\partial \operatorname{Re}[\Sigma(\omega)] / \partial \omega)^{-1}\right|_{\omega \rightarrow 0}$ (in our results $Z$ and $T_{K}$ are monotonously related with a bit weaker dependence of $Z$ on $\lambda$ ).

For any value of $\lambda$ and irrespective of its sign, the local moment is completely screened yielding a regular Fermi liquid behavior at low temperatures. Hence, one can map the low-energy excitation spectrum to that of a non-interacting resonant-level model and parametrize it in terms of the quasiparticle scattering phase shifts $\delta$. In Fig. 2(c) we present the phase shifts corresponding to the $j=1 / 2, j=3 / 2$ excitations as a function of $\lambda$. They are associated through the Friedel sum-rule to the occupancies of the corresponding resonant levels

$$
\delta_{j}=\pi n_{j} /(2 j+1) .
$$

The regime of large negative $\lambda$ corresponds to a halffilled 4-fold degenerate $j=3 / 2$ resonant level and empty $j=1 / 2$ state. Conversely, at large positive $\lambda$ the $j=3 / 2$ states are emptied out and one is left with the completely occupied $j=1 / 2$ states.

The SOC needs to be increased in absolute value above some critical value $\lambda_{c}$ in order to have any observable effect. This value, $\approx 10^{-5}$ for parameters used in this work, does not correspond to any of the bare scales of the problem but is associated to a low temperature emergent scale, the Kondo temperature. The qualitative explanation of this surprising finding is as follows: Considering the problem from the perspective of the renormaliza- 

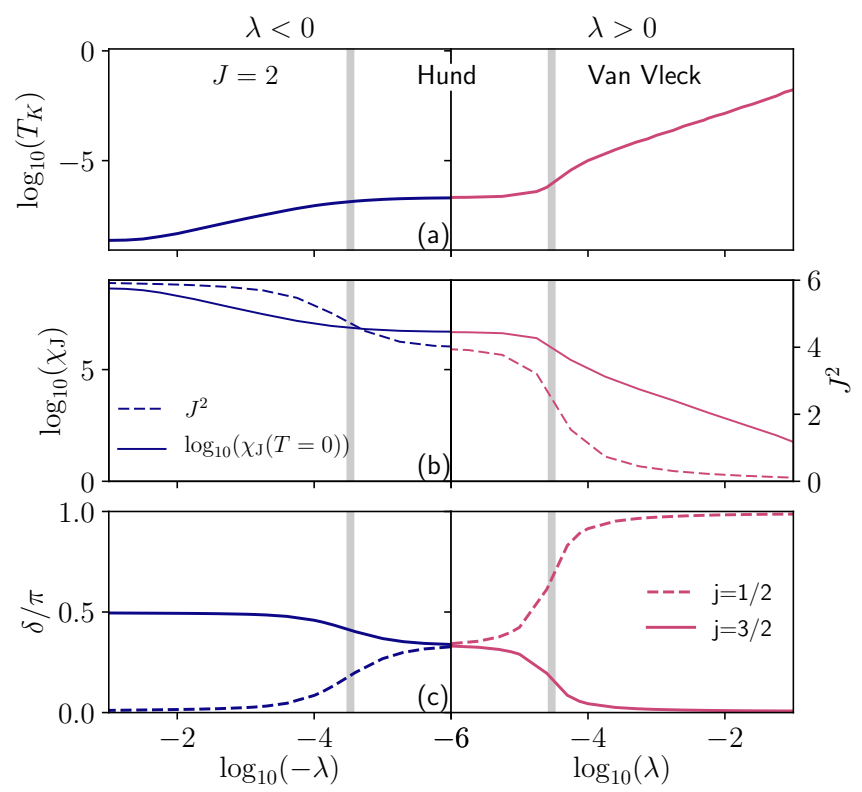

Figure 2. (a) Kondo temperature. (b) Zero-temperature total-angular-momentum susceptibility $\chi_{J}$ (full line) and expectation value $\left\langle J^{2}\right\rangle$ (dashed line). (c) Quasiparticle phase shifts.

tion group and progressively lowering the temperature, it is clear that if the moments are screened on a scale higher than $\lambda$, the SOC has nothing to act on $^{33}$. In the case of the Hund's metal, where the orbital moments are screened first, one can be more precise: $\lambda_{c}$ is determined by the onset of screening of the total angular momentum, which corresponds to the orbital Kondo temperature, which is the higher of the two Kondo screening scales. Thus $\lambda_{c} \approx T_{K}^{\text {orb }}$. This is most easily seen by inspecting the phase shifts that evolve by a half of the change to the final value at $\lambda=T_{K}^{\text {orb }}$.

We now turn to the impurity spectral functions shown in Fig. 3. For $\lambda=0$, the $j=1 / 2$ and $j=3 / 2$ excitations are degenerate, hence the corresponding spectral functions remain almost the same as long as $|\lambda|<\lambda_{c}$. One can resolve a lower Hubbard band, a broad upper Hubbard band (that contains excitations to different multiplets of half-filled impurity), and a Kondo resonance. The latter has a characteristic asymmetric shape that is a fingerprint of the Hund's metal ${ }^{34}$ (see also ${ }^{35}$ ).

When the magnitude of the spin-orbit coupling is increased, the degeneracy between the $j=1 / 2$ and the $j=3 / 2$ states is lost. In $\lambda<0$ regime the $j=1 / 2$ states are pushed to positive energies (with a low value of the spectral function at $\omega=0$, the small hump seen in the inset of Fig. 3 (a) in $j=1 / 2$ spectral function for $\lambda=10^{-3}$ is a discretization artefact); at large $|\lambda|$ this leads to a half-filled $j=3 / 2$ level with nearly symetrical lower and upper Hubbard bands. The suppression of the Kondo temperature during the crossover to $J=2$ regime is reflected in the narrowing of the Kondo resonance.

Quite different physics occurs for $\lambda>0$. The $j=1 / 2$
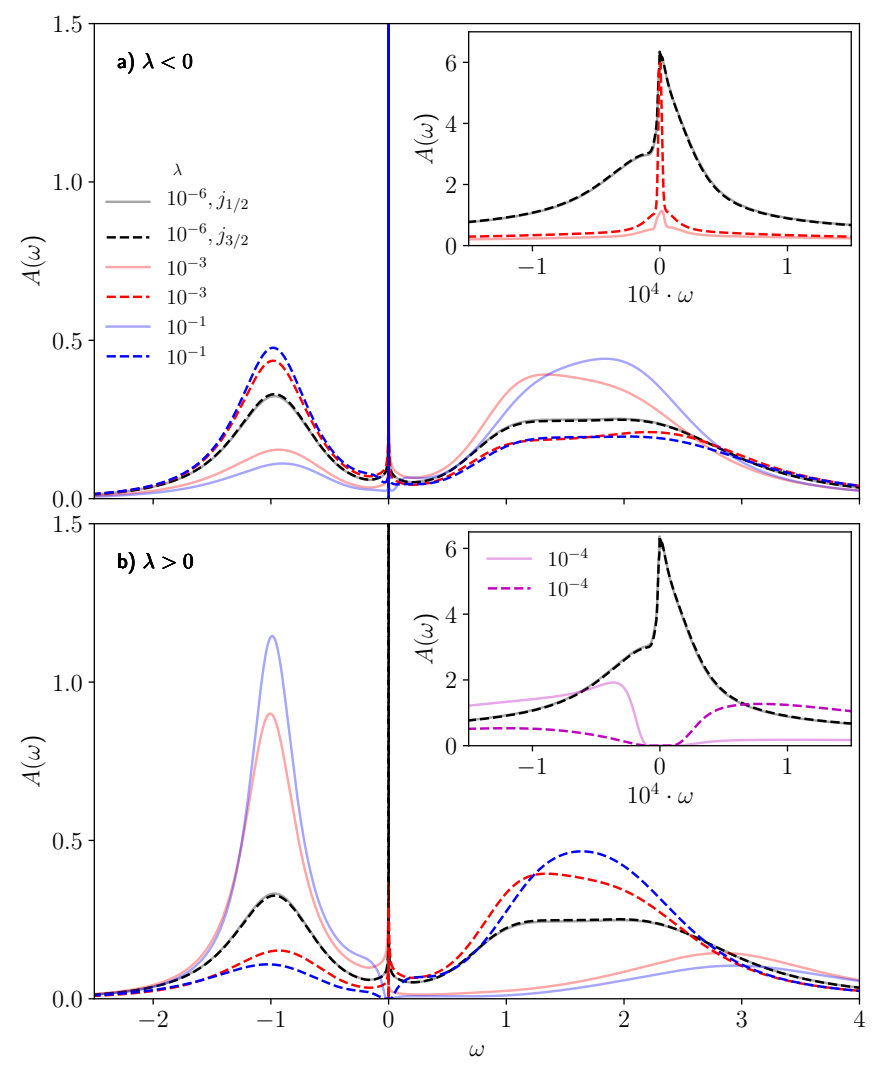

Figure 3. Impurity spectral functions in the presence of the spin-orbit coupling for $j=1 / 2$ (full lines) and $j=3 / 2$ (dashed) excitations. The inset are close-ups on the lowenergy region.

spectral weight is pushed to negative energies and the $j=3 / 2$ spectral weight to positive energies. The Kondo resonance is split by the SOC, see the inset, with the splitting being of order $\lambda$. The spectral weight at $\omega=0$ is small, an indication of the insulating-like behavior. This regime hence corresponds to that of a "non-magnetic vanVleck insulator".

\section{DISCUSSION}

It is important to note that in the context of real materials, the orbital Kondo temperature can be substantially higher than the low-temperature Fermi liquid coherence scale. In ruthenates, the orbital moments are screened at $1000 \mathrm{~K}(0.1 \mathrm{eV})^{33}$ which is similar to the estimated values of the spin-orbit coupling $(0.2 \mathrm{eV})$. This indicates that the ruthenates are within the Hund's metal regime and explains why calculations neglecting the SOC obtain reasonable results.

Our results suggest that the spin-orbit interaction could diminish the coherence scale in $d^{2}$ systems. Molybdates have such occupancy but are characterized by a coherent behavior with high Kondo temperature ${ }^{35}$ because they are far from van-Hove singularity and hence are not 
appreciably affected by the SOC. Perhaps chromates ${ }^{36}$ realize a $J=2$ metal (in spite of the smaller SOC) due to the strong correlations and hence small orbital Kondo temperature.

\section{CONCLUSION}

In summary, we investigated the effects of the SOC in a three-orbital impurity occupied by two electrons/holes that we have solved with the NRG. In $d^{2}$ systems the SOC leads to a crossover from a Hund's impurity with a distinct behavior of spin and orbital moments to a $J=2$ impurity with a suppressed Kondo temperature. In $d^{4}$ systems the crossover is to the non-magnetic regime with no local moment $(J=0)$ instead. The spectral functions in different regimes are characteristically different. The SOC becomes effective once it exceeds the emergent low-energy scale, the orbital Kondo temperature. Besides implication it has for oxides ${ }^{37}$, this finding could be tested in an STM experiment where one would control the Kondo temperature and observe the change in the shape of the spectral function as the orbital Kondo temperature falls below the SOC strength.

Acknowledgments We thank M. Aichhorn, A. Georges, A.J. Kim, M. Kim, and R. Triebl for useful discussions. We acknowledge the support of the Slovenian Research Agency (ARRS) under P1-0044.
${ }^{1}$ B. J. Kim, H. Jin, S. J. Moon, J.-Y. Kim, B.-G. Park, C. S. Leem, J. Yu, T. W. Noh, C. Kim, S.-J. Oh, J.-H. Park, V. Durairaj, G. Cao, and E. Rotenberg, Phys. Rev. Lett. 101 (2008), 10.1103/physrevlett.101.076402.

2 C. Martins, M. Aichhorn, L. Vaugier, and S. Biermann, Phys. Rev. Lett. 107, 266404 (2011).

3 R. Arita, J. Kuneš, A. V. Kozhevnikov, A. G. Eguiluz, and M. Imada, Phys. Rev. Lett. 108, 086403 (2012).

4 M. W. Haverkort, I. S. Elfimov, L. H. Tjeng, G. A. Sawatzky, and A. Damascelli, Phys. Rev. Lett. 101 (2008), 10.1103/physrevlett.101.026406.

${ }^{5}$ G.-Q. Liu, V. N. Antonov, O. Jepsen, and O. K. Andersen., Phys. Rev. Lett. 101, 026408 (2008).

${ }^{6}$ C. N. Veenstra, Z.-H. Zhu, M. Raichle, B. M. Ludbrook, A. Nicolaou, B. Slomski, G. Landolt, S. Kittaka, Y. Maeno, J. H. Dil, I. S. Elfimov, M. W. Haverkort, and A. Damascelli, Phys. Rev. Lett. 112 (2014), 10.1103/physrevlett.112.127002.

7 J. Mravlje, M. Aichhorn, T. Miyake, K. Haule, G. Kotliar, and A. Georges, Phys. Rev. Lett. 106 (2011).

8 D. Stricker, J. Mravlje, C. Berthod, R. Fittipaldi, A. Vecchione, A. Georges, and D. van der Marel, Phys. Rev. Lett. 113, 087404 (2014).

${ }^{9}$ H. T. Dang, J. Mravlje, A. Georges, and A. J. Millis, Phys. Rev. Lett. 115 (2015), 10.1103/physrevlett.115.107003.

${ }^{10}$ H. T. Dang, J. Mravlje, A. Georges, and A. J. Millis, Phys. Rev. B 91 (2015), 10.1103/physrevb.91.195149.

11 E. Gorelov, M. Karolak, T. O. Wehling, F. Lechermann, A. I. Lichtenstein, and E. Pavarini, Phys. Rev. Lett. 104, 226401 (2010).

12 A. Georges, L. de Medici, and J. Mravlje, Annu. Rev. Condens. Matter Phys. 4, 137 (2013).

13 G. Khaliullin, Phys. Rev. Lett. 111 (2013), 10.1103/physrevlett.111.197201.

14 G. Cao, T. F. Qi, L. Li, J. Terzic, S. J. Yuan, L. E. DeLong, G. Murthy, and R. K. Kaul, Phys. Rev. Lett. 112 (2014), 10.1103/physrevlett.112.056402.

15 S. Bhowal, S. Baidya, I. Dasgupta, and T. Saha-Dasgupta, Phys. Rev. B 92 (2015), 10.1103/physrevb.92.121113.

16 O. N. Meetei, W. S. Cole, M. Randeria, and N. Trivedi, Phys. Rev. B 91 (2015), 10.1103/physrevb.91.054412.

17 T. Sato, T. Shirakawa, and S. Yunoki, (2016), 1603.01800.

18 A. J. Kim, H. O. Jeschke, P. Werner, and R. Valentí, Phys. Rev. Lett. 118, 086401 (2017).
19 A. A. Khajetoorians, M. Valentyuk, M. Steinbrecher, T. Schlenk, A. Shick, J. Kolorenc, A. I. Lichtenstein, T. O. Wehling, R. Wiesendanger, and J. Wiebe, Nature Nanotech 10, 958 (2015).

20 A. A. Khajetoorians, M. Steinbrecher, M. Ternes, M. Bouhassoune, M. dos Santos Dias, S. Lounis, J. Wiebe, and R. Wiesendanger, Nature Communications 7, 10620 (2016).

21 D.-J. Choi, S. Guissart, M. Ormaza, N. Bachellier, O. Bengone, P. Simon, and L. Limot, Nano Lett. 16, 6298 (2016).

22 S. Sugano, Multiplets of Transition-Metal Ions in Crystals (Academic Press, 1970).

23 R. Bulla, T. A. Costi, and T. Pruschke, Rev. Mod. Phys. 80, 395 (2008).

24 R. Žitko, "NRG Ljubljana," nrgljubljana.ijs.si/.

25 W. C. Oliveira and L. N. Oliveira, Phys. Rev. B 49, 11986 (1994).

26 V. L. Campo and L. N. Oliveira, Phys. Rev. B 72, 104432 (2005).

27 R. itko, Computer Physics Communications 180, 1271 (2009).

28 R. Peters, T. Pruschke, and F. B. Anders, Phys. Rev. B 74, 245114 (2006).

29 Z. P. Yin, K. Haule, and G. Kotliar, Nat. Mater. 10, 932 (2011).

30 C. Aron and G. Kotliar, Phys. Rev. B 91 (2015), 10.1103/physrevb.91.041110.

31 A. Horvat, R. Žitko, and J. Mravlje, Phys. Rev. B 94, 165140 (2016).

32 One needs to be careful in interpreting $T_{K}$ as a Kondo temperature in this regime as the quenching of the moment is not due to conduction electrons but is rather atomic.

33 J. Mravlje and A. Georges, Phys. Rev. Lett. 117, 036401 (2016).

${ }^{34}$ K. M. Stadler, Z. P. Yin, J. von Delft, G. Kotliar, and A. Weichselbaum, Phys. Rev. Lett. 115 (2015), 10.1103/physrevlett.115.136401.

${ }^{35}$ H. Wadati, J. Mravlje, K. Yoshimatsu, H. Kumigashira, M. Oshima, T. Sugiyama, E. Ikenaga, A. Fujimori, A. Georges, A. Radetinac, K. S. Takahashi, M. Kawasaki, and Y. Tokura, Phys. Rev. B 90, 205131 (2014).

${ }^{36}$ L. de' Medici, J. Mravlje, and A. Georges, Phys. Rev. Lett. 107, 256401 (2011). 
37 See also a very recent preprint, M. Kim et al. arXiv:1707.02462 for a DMFT study that finds behavior fully consistent with the one found in the impurity model here. 